\title{
Non-linear electrical conduction and broad band noise in the charge-ordered rare earth manganate $\mathrm{Nd}_{0.5} \mathrm{Ca}_{0.5} \mathrm{MnO}_{3}$
}

\author{
Ayan Guha*, Arindam Ghosh, A.K.Raychaudhuri \\ Department of Physics, Indian Institute of Science, Bangalore 560 012, India \\ S. Parashar, A.R.Raju, C.N.R.Rao \\ Jawaharlal Nehru Centre for Advanced Scientific Research, Jakkur P.O., Bangalore 560 064, India

\begin{abstract}
Measurements of the dc transport properties and the low-frequency conductivity noise in films of charge ordered $\mathrm{Nd}_{0.5} \mathrm{Ca}_{0.5} \mathrm{MnO}_{3}$ grown on Si subtrate reveal the existence of a threshold field in the charge ordered regime beyond which strong non linear conduction sets in along with a large broad band conductivity noise. Threshold-dependent conduction disappears as $T \rightarrow T_{C O}$, the charge ordering temperature. This observation suggests that the charge ordered state gets depinned at the onset of the non-linear conduction.
\end{abstract}

Rare-earth manganites with a general chemical formula $\mathrm{Re}_{1-x} \mathrm{Ae}_{x} \mathrm{MnO}_{3}$ (where Re is a trivalent rare-earth and Ae is a divalent alkaline earth cation) show a number of interesting phenomena like Colossal Magnetoresistance (CMR) and Charge Ordering (CO) [1]. These compounds belong to the $\mathrm{ABO}_{3}$ type perovskite oxides where Re and Ae ions occupy the A site and Mn occupies the B site. It has been known for some time that these manganites (depending on the size of the average A-site cationic radius $\left\langle r_{A}\right\rangle$ ) can charge order, for certain values of $\mathrm{x}$. The nature of the $\mathrm{CO}$ state depends on the value of $\left\langle r_{A}\right\rangle$ and it is stabilized if the value of $<r_{A}>$ is smaller. The $\mathrm{CO}$ transition is associated with a lattice distortion as well as orbital and spin ordering.

Recent experiments have established that CO state is strongly destabilized by a number of different types of perturbations. An applied magnetic field of sufficient magnitude can lead to a collapse of the CO gap $\Delta_{C O}$ and melting of the $\mathrm{CO}$ state [2, 3]. The $\mathrm{CO}$ phenomenon is stabilized by lattice distortion. A perturbation to the distortion can also destabilize the $\mathrm{CO}$ state [П]. Recently it has been reported that application of an electric field [5].6], optical radiation [7], or x-ray radiation [8] melts the CO state in $\operatorname{Pr}_{0.7} \mathrm{Ca}_{0.3} \mathrm{MnO}_{3}$. It is not clear, however, what causes destabilization of the $\mathrm{CO}$ state in these cases and whether the underlying mechanism is same for all perturbations.

Electric field induced melting of the CO state leads to a strong non-linear conduction as seen in the bulk [5] as well as in films [6]. This raises a very important question whether there is a threshold field associated with the non-linear conduction. In a driven system pinned by a periodic potential there exists a threshold force beyond which the system is depinned [9]. If the system is charged and the driving force comes from an electric field then this shows as a threshold field or bias for the onset of a non-linear conduction.Existence of a threshold field would imply that the melting of the $\mathrm{CO}$ state by an applied electric field can actually be a depining phenomena. We investigated this in films of the $\mathrm{CO}$ system $\mathrm{Nd}_{0.5} \mathrm{Ca}_{0.5} \mathrm{MnO}_{3}$ by careful measurement of field dependent dc transport at various temperatures and also followed it up with a measurement of electrical noise (voltage fluctuation) as a function of applied dc bias. We made the following important observations :

(1) There indeed exists a threshold field $\left(E_{t h}\right)$ below the CO temperature $T_{C O}$ and for $E>E_{t h}$ a strong nonlinear conduction sets in.

(2) $E_{t h}$ strongly depends on $T$ and $E_{t h} \rightarrow 0$ as $T \rightarrow T_{C O}$. (3) For $T<T_{C O}$, a large voltage fluctuation $\left(<\delta V^{2}>/ V^{2}\right)$ appears at the threshold field. Both $E_{t h}$ and $<\delta V^{2}>/ V^{2}$ reaches a maximum at $T \approx$ $90 \mathrm{~K}\left(\approx 0.4 T_{C O}\right)$.

(4) The spectral power distribution of the voltage fluctuation is broad band and has nearly $1 / \mathrm{f}$ character.

In $\mathrm{Nd}_{0.5} \mathrm{Ca}_{0.5} \mathrm{MnO}_{3}$, a system with relatively small $\left.<r_{A}\right\rangle$, the CO transition takes place from a high temperature charge disordered insulating phase to a charge ordered insulating phase (COI). Charge ordering in this system has been studied by us in details previously [10]. Poly-crystalline films of $\mathrm{Nd}_{0.5} \mathrm{Ca}_{0.5} \mathrm{MnO}_{3}$ (average thickness $\approx 1000 \mathrm{~nm}$ ) were deposited on $\mathrm{Si}(100)$ single crystal substrates by nebulized spray pyrolysis of organometallic compounds. The details of sample preparation and characterisation (including X-ray) have been given elsewhere [6]. Contacts were made by sputtering gold on the films and connecting the current and voltage leads on the gold contacts by silver paint. The I-V characteristics was measured by dc current biasing and the voltage between the voltage leads was measured by a nano-voltmeter . For measuring the electrical noise, the fluctuating component of the voltage $\delta V$ was amplified by $5 \times 10^{3}$ times by a low noise pre-amplifier. The output of the pre-amplifier was sampled by an ADC card and the data were directly transferred to the computer. The temperature was controlled to within $10 \mathrm{mK}$ for both the 
measurements.

The films have a $T_{C O} \approx 250 \mathrm{~K}$ as seen from the resistivity data.The resistivity was measured at a measuring current of $3 \mathrm{nA}$, which is much lower than the current where non-linear conductivity sets in. The experiment was conducted down to $80 \mathrm{~K}$ where the sample resistance becomes more than $100 \mathrm{M} \Omega$, the limit of our detection electronics.

In figure 1, we show the typical I-V curves at few characteristic temperatures.At all the temperatures (except that at $220 \mathrm{~K}$ ) there is a clear signature of a threshold voltage $V_{t h}$ beyond which the current rises significantly signalling the onset of strong nonlinear conduction. (The separation of electrodes in our experiment is $2 \mathrm{~mm}$, so that $E_{t h}=5 V_{t h}$ volts $\left./ \mathrm{cm}\right)$. I-V curves show two components of conduction: a normal component which exists at all $\mathrm{V}$ and a strongly non-linear component starting at $V>V_{t h}$. The normal component although not exactly linear in I-V, has much less non linearity. We fit our I-V data using the following empirical expression which allows us to separate out the two components :

$$
I=f_{1}\left(V-V_{t h}\right)+f_{2}(V)=C_{1}\left(V-V_{t h}\right)^{n_{1}}+C_{2} V^{n_{2}}
$$

where $f_{1}$, a function of $\left(V-V_{t h}\right)$, is the component of current that has a threshold associated with it and $C_{1}=0$ for $V<V_{t h}$. The component $f_{2}$ is the normal conduction component. $C_{1}, C_{2}, n_{1}, n_{2}$ are constants for a given temperature. The data at all temperatures can be well fitted to eqn.1 for $T>90 \mathrm{~K}$ as shown by the solid lines in figure 1. The dashed and dashed-dotted lines give the contributions of each of the terms. For $T<90 \mathrm{~K}$ certain additional features show up (see data at $81 \mathrm{~K}$ ) in the $\mathrm{I}-\mathrm{V}$ data which give impression that there may be multiple thresholds. In figure 2(a) we have plotted the threshold voltage $V_{t h}$ as a function of $T$ as obtained from eqn.1. It can be seen that $V_{t h} \rightarrow 0$ as $T \rightarrow T_{C O}$. Within the limitations of our detectibility, we could see a finite nonzero $V_{t h}$ upto $T \approx 170 K \approx 0.7 T_{C O}$. Beyond this temperature it is difficult to distinguish between the two conduction components.

The relative contributions of $f_{1}$ and $f_{2}$ to the total current (expressed as the ratio $f_{1} / f_{2}$ evaluated at $I=1 \mu A$ ) has been plotted as a function of $\mathrm{T}$ in figure $2(\mathrm{~b})$. At $\mathrm{T}_{\mathrm{ii}} \mathrm{T}_{C O}$, the non-linear component is orders of magnitude larger than the normal conduction component and they are comparable as $\mathrm{T} \rightarrow T_{C O}$. The exponent $n_{1}$ is strongly temperature dependent and from a value $\approx 2$ at $160 \mathrm{~K}$ it reaches a value more than 5 at $T \approx 100 \mathrm{~K}$. The exponent $n_{2}$ does not have much of a temperature dependence and is $\approx 1.1-1.4$ for $\mathrm{T} \leq 180 K$.
In pinned driven system one often sees onset of broad band noise as the system is depinned at the threshold voltage [9. We find that such is indeed the case in this system. In figure 3 we show the magnitude of the voltage fluctuation $<\Delta V^{2}>/ V^{2}$ as a function of the applied bias $\mathrm{V}$ at $T=100 \mathrm{~K}$ along with the $\mathrm{I}-\mathrm{V}$ curve. The arrow indicates $V_{t h}$. It is clear that the voltage fluctuation has a non monotonous dependence on $\mathrm{V}$ and reaches a peak at $V \approx V_{t h}$. This fluctuation has been seen at all $T<0.7 T_{C O}$ where we can detect measurable $V_{t h}$. The peak values of the fluctuation measured at different $\mathrm{T}$ are shown in figure $2(\mathrm{c})$. The fluctuation $\rightarrow 0$ as $T \rightarrow T_{C O}$ and has a peak at $90 \mathrm{~K}$ where $V_{t h}$ also shows a peak.

Frequency dependences of the spectral power $S_{V}(f)$ measured at $100 \mathrm{~K}$ with biases $\mathrm{V}_{i} \mathrm{~V}_{t h}, \mathrm{~V} \approx \mathrm{V}_{t h}$ and $\mathrm{V}_{i} \mathrm{~V}_{t h}$ are shown in figure 4 . We have plotted the data as $f . S_{V} / V^{2}$ vs. $f$. For a pure $1 /$ f noise $\left(S_{V} \propto 1 / \mathrm{f}\right)$, this should be a straight line parallel to the f-axis. It can be seen that the predominant contribution to noise has $1 / \mathrm{f}$ character.In addition,there is another broad band contribution riding on the main $1 / \mathrm{f}$ contribution. At higher $\mathrm{V}$ the spectra becomes more of $1 / \mathrm{f}$ nature.

The onset of strong non-linear conduction at a threshold voltage and the accompanied broad band noise has been seen in solids like $\mathrm{NbSe}_{3}, \mathrm{TaS}_{3}$ which show depinning of charge density waves (CDW) by a threshold field [9]. Though the physics of CDW and CO states are entirely different, the underlying phenomenological description of depining can be similar. Electron diffraction (ED) and electron microscopy studies on a CO system $\left(\mathrm{La}_{0.5} \mathrm{Ca}_{0.5} \mathrm{MnO}_{3}\right)$ have shown that the $\mathrm{CO}$ is associated with formation of stable pairs of $\mathrm{Mn}^{3+} \mathrm{O}_{6}$ stripes. The $\mathrm{Mn}^{3+} \mathrm{O}_{6}$ octahedra in the stripes are strongly distorted by the Jahn-Teller (JT) distortion [11].It is possible that the stability of the $\mathrm{CO}$ system depends on the stability of the stripes which can be pinned. The strong JT distorted pairs of the $\mathrm{Mn}^{3+} \mathrm{O}_{6}$ octahedra can act as periodic pinning sites due to local strain field. From our data for $T<90 \mathrm{~K}$, it seems there are changes occuring below $90 \mathrm{~K}$. We are not clear about the changes. We only note that in magnetic studies we found that strong irreversibility sets in below $80 \mathrm{~K} 10$.

To conclude, the present study demonstrates that there is a threshold field associated with the onset of non-linear conduction in the $\mathrm{CO}$ system along with the existence of a broad band noise. The observation is taken as evidence of depinning of $\mathrm{CO}$ state as the origin of non-linear conduction in these solids. 
[1] Colossal Magnetoresistance, Charge Ordering and Related Properties of Manganese Oxides, edited by C.N.R. Rao and B. Raveau (World Scientific, Singapore, 1998).

[2] H. Kuwahara, Y. Tomioka, A. Asamitsu, Y. Moritomo, and Y. Tokura Science 270, 961 (1995).

[3] Amlan Biswas, Anthony Arulraj, A.K. Raychaudhuri, C.N.R. Rao preprint cond-mat / 9903110.

[4] A. Arulraj, A. Biswas, A.K. Raychaudhuri, C.N.R. Rao, P.M. Woodward, T. Vogt, D.E. Cox, A.K. Cheetham, Phys. Rev. B (Rapid com). 57, R8115 (1998).

[5] A. Asamitsu, Y. Tomioka, H. Kuwahara, and Y. Tokura, Nature (London) 388, 50 (1997).

[6] V. Ponnambalam, Sachin Parashar, A.R.Raju, and C.N.R. Rao Appl. Phys. Lett.74, 206 (1999).

[7] K. Ogawa, W.Wei, K. Miyano, T. Tomiyoka, and Y.Tokura, Phys Rev. B 53, R15033 (1998); M. Fiebig, K. Miyano, Y. Tomiyoka, and Y. Tokura, Science 280, 1925 (1998).

[8] V. Kiryukhin, D. Casa, J.P. Hill, B. Keimer, A. Vigliante, Y. Tomioka, and Y. Tokura, Nature (London) 386, 813 (1997).

[9] G. Gruner, Rev. Mod. Phys. 60, 1129 (1988).

[10] R.Mahendiran, R.Mahesh, R.Gundakaram, A.K.Raychaudhuri and C.N.R. Rao, J. Phys : Condens. Matter (Lett) 8, L455, (1996). T. Vogt, A.K. Cheetham, R. Mahendiran, A.K. Raychaudhuri, R. Mahesh, C.N.R. Rao, Phys Rev B 54, 14926 (1996).

[11] S. Mori, C.H. Chen, S.W. Cheong, Nature (London) 392, 473 (1998).

\section{FIGURE CAPTIONS}

(1) FIG. 1. I-V curves at different temperatures, solid line shows the total I, dashed and dashed-dotted lines show the components $\mathrm{f}_{1}$ and $\mathrm{f}_{2}$.

(2) FIG. 2 Temperature variation of (a) resistivity, (b) magnitude of threshold voltage, (c) relative contributions of $f_{1}$ and $f_{2}$ and $(d)$ noise magnitude at the threshold voltage

(3) FIG. 3 The noise magnitude and I-V characteristics at $100 \mathrm{~K}$. The arrow indicates the threshold voltage.

(4) FIG. 4 Frequency spectrum of the noise at $100 \mathrm{~K}$ for different bias values. 


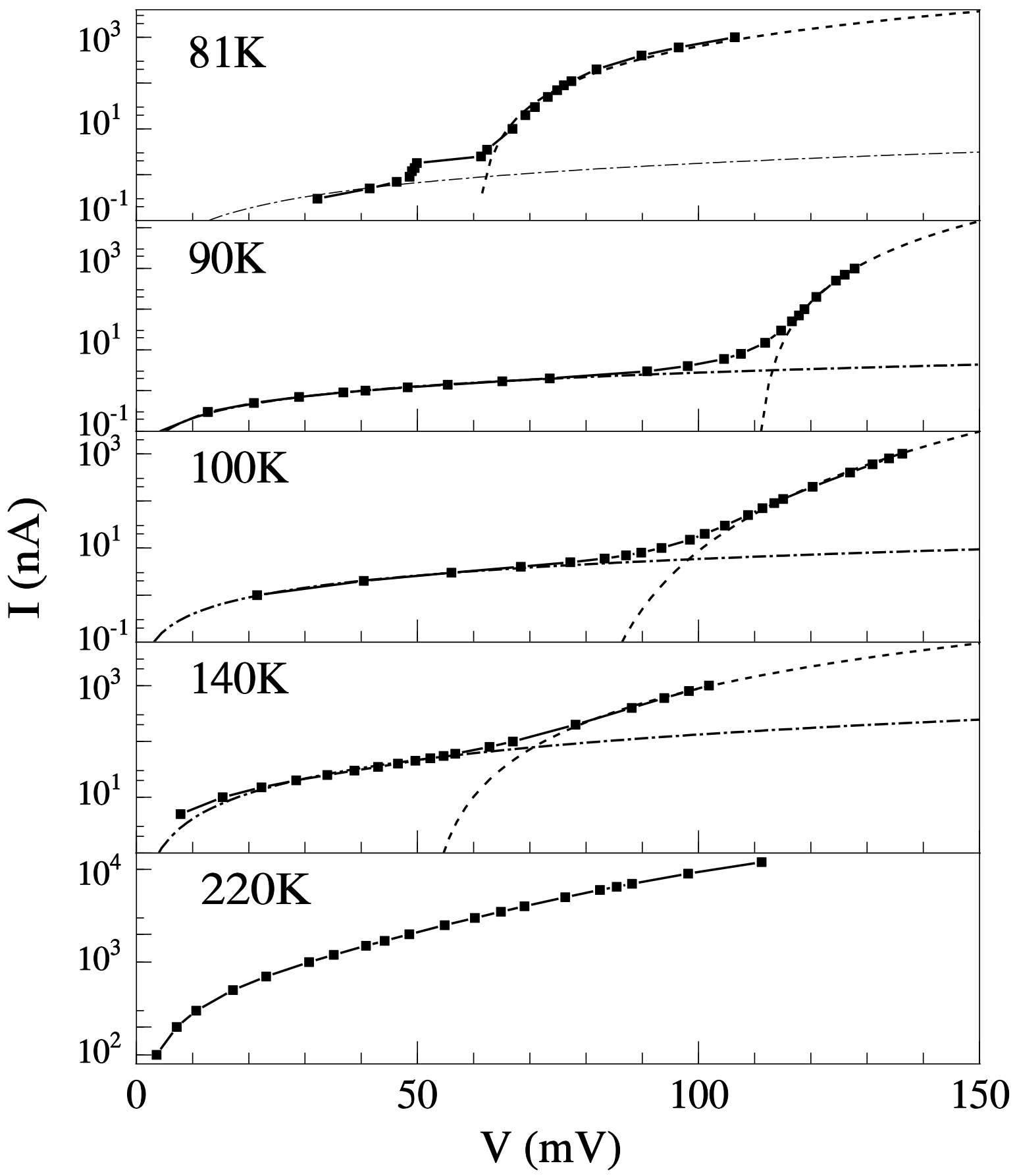




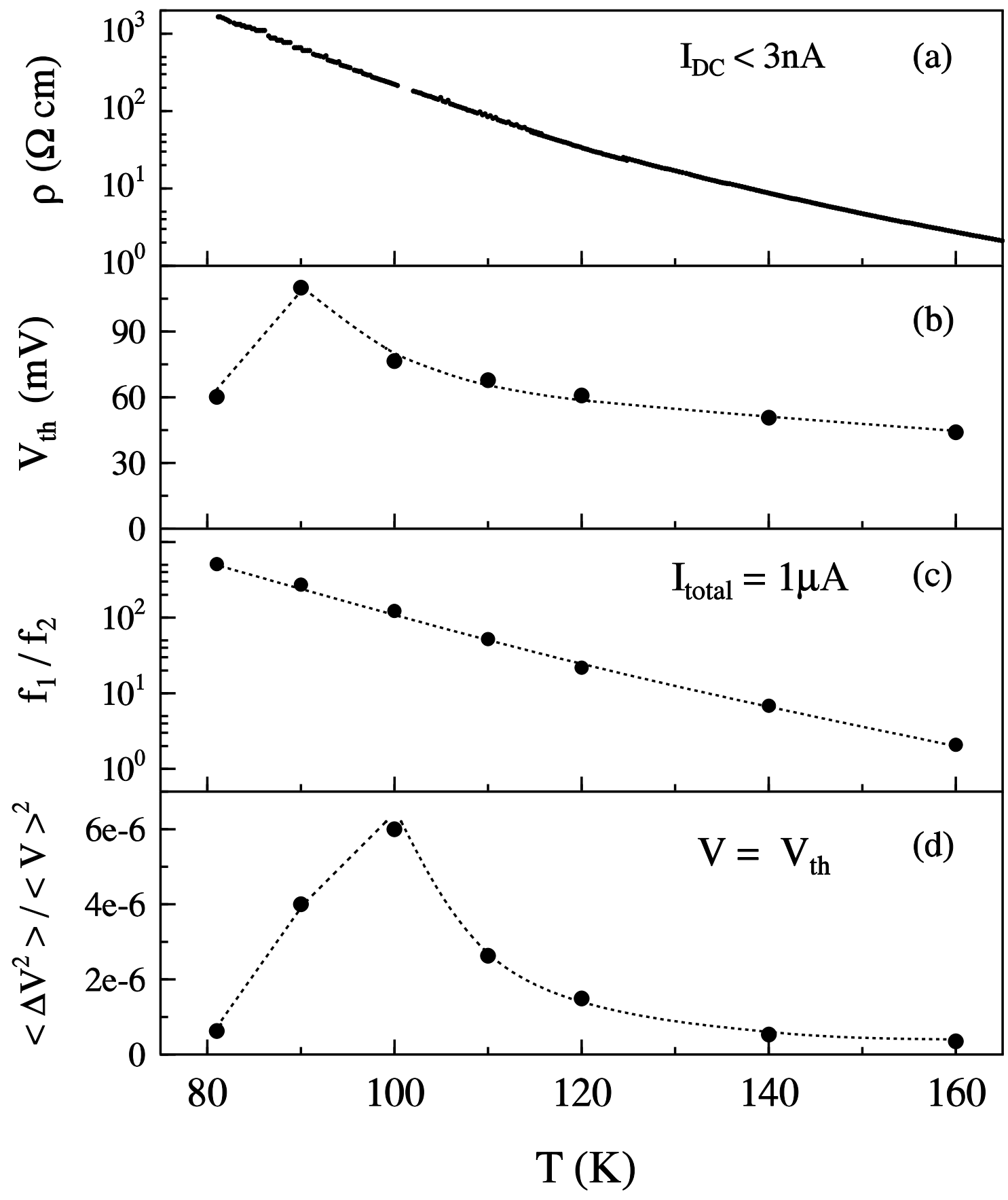




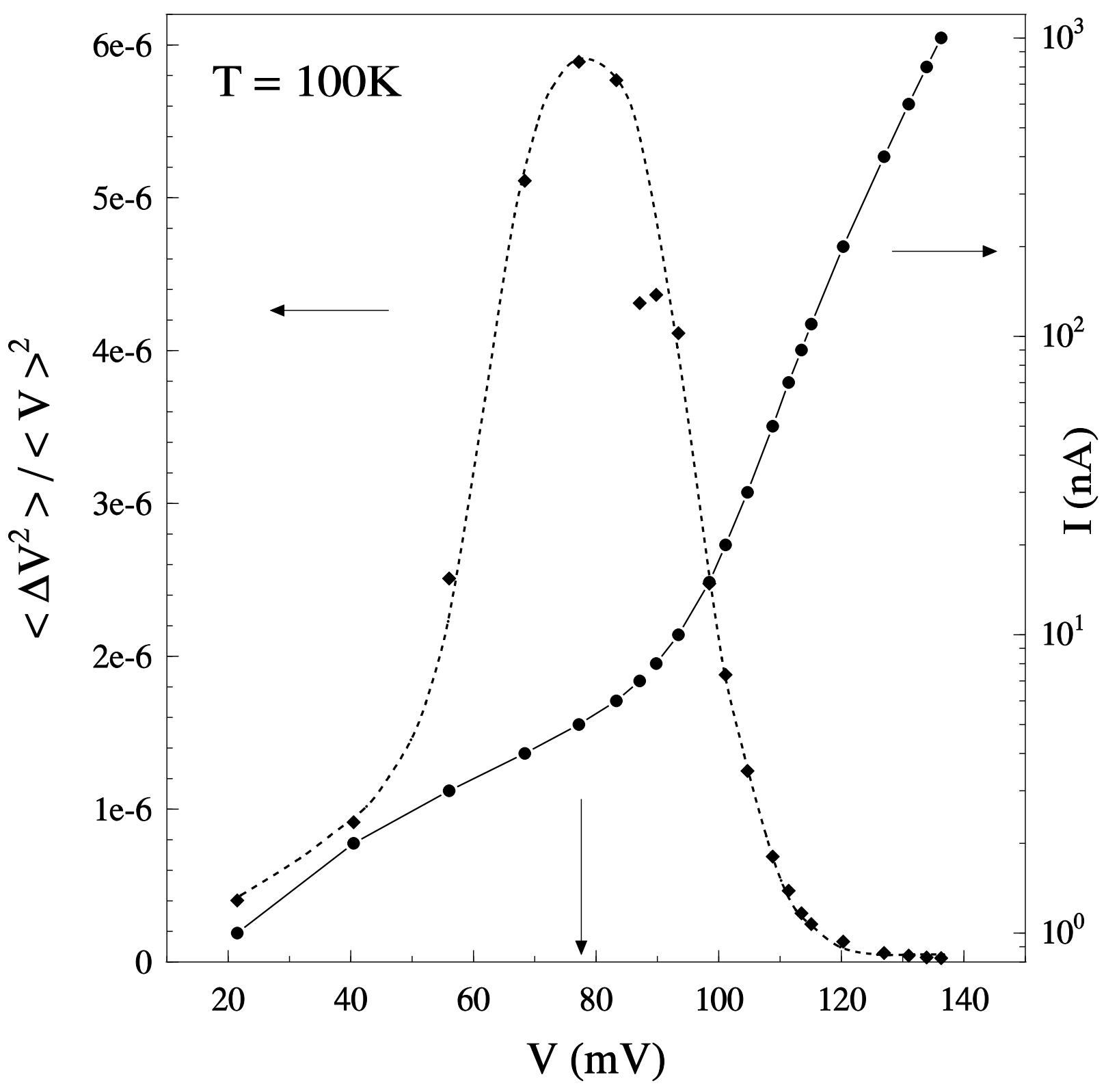




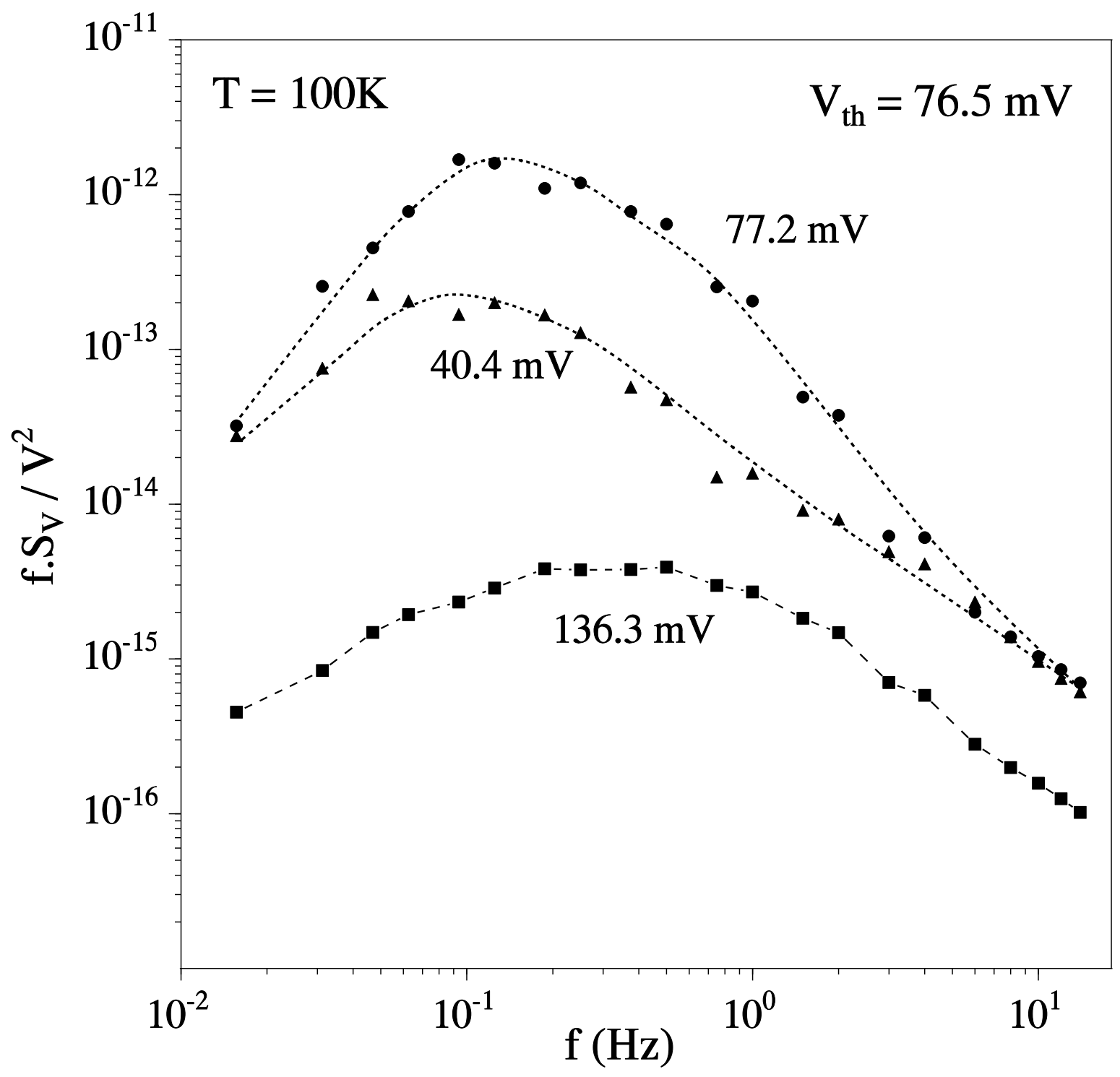

